\title{
Vestibular system dysfunction in patients after mild traumatic brain injury
}

\author{
Wojciech Skóra' ${ }^{1, A-F}$, Ramzes Stańczyk ${ }^{2, C, E}$, Anna Pajor ${ }^{2, A, C, E}$, Magdalena Jozefowicz-Korczyńska ${ }^{2, A-F}$ \\ ${ }^{1}$ Otolaryngology Department, S. Rybicki Memorial Regional Hospital in Skierniewice, Poland \\ 2 Balance Disorder Unit, Otolaryngology Department, Medical University of Lodz, Norbert Barlicki Memorial University \\ Hospital No. 1, Poland \\ A - Research concept and design, B - Collection and/or assembly of data, C - Data analysis and interpretation, \\ $D$ - Writing the article, E - Critical revision of the article, F - Final approval of article
}

Skóra W, Stańczyk R, Pajor A, Jozefowicz-Korczyńska M. Vestibular system dysfunction in patients after mild traumatic brain injury. Ann Agric Environ Med. 2018; 25(4): 665-668. doi: 10.26444/aaem/81138

\begin{abstract}
Introduction. In the literature there are some discrepancies concerning the occurrence of vertigo and disequilibrium, as well as the type of vestibular dysfunction in patients after mild traumatic brain injury (MTBI).

Objective. The aim of the study was to assess the type of vestibular system dysfunction in MTBI patients after injury at baseline and 6-month follow-up.

Materials and method. From a group of 188 patients with vertigo/dizziness and balance instability after MTBI, prospective analysis in 50 patients with abnormal videonystagmography (VNG) were conducted. Anamnesis, otoneurological examination, self-assessed Dizziness Handicap Inventory (DHI) and VNG test were carried in each patient twice - at baseline and after 6 months.

Results. A significant improvement in the DHI total score $(9.8$ points, $\mathrm{p}<0.001)$ and in the subscales was found after 6 months. At baseline, VNG test showed the peripheral vestibular system dysfunction in 19 cases (38\%), central vestibular dysfunction in 20 patients (40\%) and mixed vestibular dysfunction in 11 cases (44\%). After 6 months, the VNG records normalization was observed in 19 patients (38\%), an improvement was significantly more frequently related to peripheral disorders, compared to central ones $(p<0.001)$.

Conclusions. In patients after mild traumatic brain injury, about one-fourth of them had vestibular system dysfunction with the same frequency of peripheral, central and mixed lesions in videonystagmography. Subjective post-traumatic symptoms and vestibular system dysfunction in patients after MTBI decrease after 6-month follow-up. However, more than half of patients still have abnormal VNG results, which indicate dysfunction of the central vestibular system.
\end{abstract}

\section{- Key words}

vertigo, dizziness, mild traumatic brain injury, disequilibrium

\section{INTRODUCTION}

The continuous development of civilization and an associated rapid growth in the incidence of motor crashes and ensuing injuries are the reason why general practitioners, laryngologists or neurologists more often consult the victims of those accidents who are suffering from posttraumatic vertigo or disequilibrium. The number of traumatic injuries is increasing immensely in industrialized countries and mild traumatic brain injury (MTBI) is one of the most common types [1-5]. Patients after severe head injuries are often referred to the ambulatory emergency care units and those with mild traumas seek medical care from family physicians or general practitioners at outpatient departments or speciality healthcare centres at various times after injury. However, according to Nygren-de Boussard et al. [6], some patients with MTBI do not visit a physician at all. The diagnostic criteria for MTBI established by the American Congress of Rehabilitation Medicine include loss of consciousness, chronic headache, dizziness, feeling dazed or confused, the occurrence of post-traumatic memory disturbances, and

Address for correspondence: Wojciech Skóra, Otolaryngology Department, S. Rybicki Memorial Regional Hospital in Skierniewice, ul. Rybickiego 1 , 96-100 Skierniewice, Poland

E-mail:wojciech.skora@centrumlaryngologii.pl

Received: 07.10.2017; accepted: 11.12.2017; first published: 14.02.2018 cognitive or emotional disorders arising from experienced injuries [7].

The main causes of head injuries are traffic accidents, incidents during sports and leisure activities or accidents at work. During multi-organ injuries, the head is the region of the human body most susceptible to trauma. Such injuries appear to be a direct cause of up to $50 \%$ of post-traumatic deaths and most cases of permanent injury-related disabilities $[1,4,8]$. Epidemiological data on the incidence of head injuries are difficult to determine in different countries on the basis of routinely collected data. In the United States, it is estimated that 1.5-2 million people sustain head trauma annually, of which 400,000 require hospitalization. However, many injuries are not included in the statistics because patients do not always seek help [1, 2, 4-8].

'Post-traumatic dizziness/vertigo' is a term that indicates a common etiology for a very heterogeneous presentation of peripheral and central vestibular system dysfunction. Patients with benign positional paroxysmal vertigo (BBPV) as a result of a traumatic event constitute a different diagnostic and treatment group [9-11]. In literature, there are quite divergent data on the incidence of imbalance and dizziness after head traumas. This incidence, according to the results of studies reported by various researchers, varies from $14 \%$ - 20-90\% for non-hospitalized patients, depending on the established criteria $[12,13]$. However, the incidence rate 
of abnormal results in objective vestibular function tests in such patients is in the range of $32-61 \%[13,14]$. The duration of post-traumatic dysfunction of the balance system is varied. In patients after head injury, dizziness or vertigo persists from one week to several years [15-20]. In the case of mild head injury, the symptoms of the post-concussion syndrome, including those of the balance system, may last for a year or even longer in $10-15 \%$ of patients, and this percentage increases to $28 \%$ in moderate traumatic injuries $[1,21]$. Dizziness after brain injury is also a risk factor for a prolonged recovery [22].

\section{OBJECTIVE}

There are only a few research papers in the literature that present the divergent data on vestibular system impairment after head traumas. Most of these studies evaluated authors' diagnostic protocol without subsequent follow-up observations. Consequently, the aim of this study was to analyse the type of vestibular system dysfunction in MTBI patients with persisting dizziness and/or imbalance, and assess a 6-month follow-up.

\section{MATERIALS AND METHOD}

Fifty patients (26.6\%) from a group of 188 subjects who were diagnosed for vertigo/dizziness and balance instability after MTBI at the Balance Disorder Unit, Otolaryngology Department, Medical University in Lodz, Poland, were subject to a prospective analysis. The study design was approved by the Bioethics Committee of the Medical University in Lodz (No. RNN/87/12/KE).

Patients after MTBI, 1 - 2 months prior to the study, with persistent dizziness and/or imbalance symptoms, meeting the criteria of the World Health Organization (WHO) of 2004 [25] were included in the study. According to these criteria, MTBI diagnosis should be based on the occurrence of at least one of the 4 following elements in the patients not requiring surgical treatment:

1) loss of consciousness for not more than 30 minutes;

2) posttraumatic amnesia not longer than 24 hours;

3) any alteration in mental state (feeling dazed or disoriented);

4) any episode of dizziness and disequilibrium; or transient neurological disorders (focal, paroxysmal symptoms).

Patients with normal results of videonystagmography (VNG) and those with a prior history of any balance or ear disorders were excluded from the study.

The study group of 50 patients after MTBI with persistent symptoms of dizziness / disequilibrium lasting for more than a month, comprised 22 women and 28 men, aged $16-77$ years (mean age: $44.4 \pm 13.6$ years). All patients were interviewed and completed a questionnaire which included information on the type and circumstances of the injury, ailments, loss of consciousness and general state of health and lifestyle. All subjects also completed the Dizziness Handicap Inventory (DHI), a 25-question instrument used for self-assessment of disability due to dizziness. It is divided into 3 components that assess the physical (e.g., Does looking up increase your problem?), emotional (e.g., Because of your problem, do you feel frustrated?) and functional (e.g., Because of your problem, do you have difficulty getting into or out of bed?) person's symptoms. A total possible score of 100 is the result of 36 points from the emotional scale ( 9 items), 36 points from the functional scale ( 9 items) and 28 points from the physical scale (7 items). Answers are graded 0 (never), 2 (sometimes), and 4 (always). Higher scores indicate a greater degree of impairment. The result in the range of $0-39$ points manifest a mild form of the disorder, 40 - 69 points - a moderate form, and 70 - 100 points - a severe form of the disorder [23].

The vestibular system was evaluated based on the results of videonystagmography VNG (Ulmer SYNAPSIS 2008) in all patients. The VNG examination assessed spontaneous ocular movements with eyes open and closed, positional tests, ocular-motor tests - smooth pursuit, optokinetic and saccadic tests, kinetic stimulation with torsion swing test, and caloric test by the Fitzgerald-Hallpike method. The results were determined as peripheral, central or both (mixed) impairments. Clinical and VNG examinations as well as DHI were conducted twice: at the baseline visit and 6-month follow-up visit.

Statistical analysis was performed by Statistica version 6.0 Software. The results are expressed as means with standard deviation (SD) and data were checked for normality by the Shapiro-Wilk. Comparisons between the baseline examination and the 6-month follow-up were carried out using the Student's t-test, and the Wilcoxon's test was applied when the test assumptions were not met. For parameters presented in a nominal scale, the frequency of occurrence of the classes concerned was calculated. Frequency comparisons were performed with the $\chi 2$ test, and the Fisher exact test was used if the test assumptions were not met. Values of $\mathrm{p}<0.05$ were accepted as significant.

\section{RESULTS}

The most common causes of injury were traffic accidents - 16 patients (32\%), accidents at home or those occurring as a result of robbery/assault - 10 patients (20\%), during sports events -9 patients (18\%), and accidents at work -4 patients (8\%).

Dizziness occurred immediately after injury in 28 patients (56\%), while in 22 patients (44\%) after a few days to several weeks. At the baseline examination, patients also reported ailments associated with the balance system, such as walking instability - 23 patients (46\%), non-systemic panic attacks 21 people (42\%), static balance impairment -20 people (40\%). Short-term loss of consciousness related to trauma occurred in 23 patients (46\%) and lasted less than 30 minutes in all cases. At the follow-up, 6 months after the traumatic injury, the patients most frequently reported non-systemic dizziness - 12 subjects (24\%), followed by walking disorders - 9 subjects (18\%), static imbalance -6 subjects $(12 \%)$, and paroxysmal dizziness -3 subjects (6\%). There were no patients with concomitant vegetative disorders. A statistically significant decrease in the incidence of all equilibrium disorders was found ( $\mathrm{p}<0.05)$.

Comparing the results of subjective assessment with the use of the DHI questionnaire during the first (baseline) and second examination 6 months after the injury, the most significant difference was found in the total scoring, i.e. in a decrease by 9.8 points $(\mathrm{p}<0.001)$. Smaller differences, although also statistically significant $(\mathrm{p}<0.001)$, were observed in the 
Table 1. Mean results of Dizziness Handicap Inventory (DHI) questionnaire in the group of patients after mild traumatic brain injury at baseline visit and 6-month follow-up control visit $(n=50)$

\begin{tabular}{lccc}
\hline DHI & $\begin{array}{c}\text { Baseline visit } \\
\text { mean (points) } \\
\pm \text { SD }\end{array}$ & $\begin{array}{c}\text { Control visit } \\
\text { mean (points) } \\
\pm \text { SD }\end{array}$ & $\begin{array}{c}\text { Differences } \\
\text { (points) }\end{array}$ \\
\hline Total score & $37.6 \pm 22.2$ & $27.8 \pm 23.1^{*}$ & 9.8 \\
\hline Subscale P- physical & $13.1 \pm 8.0$ & $9.9 \pm 8.1^{*}$ & 3.2 \\
\hline Subscale E- emotional & $11.1 \pm 8.3$ & $8.4 \pm 8.3^{*}$ & 2.7 \\
\hline Subscale F- functional & $13.4 \pm 8.2$ & $9.6 \pm 8.6^{*}$ & 3.8 \\
\hline${ }^{*}<<0.001$ & & &
\end{tabular}

functional (-3.8 points), physical (-3.2 points) and emotional (-2.7 points) subscales (Tab. 1). After the 6-month follow-up, an improvement in DHI scoring was detected in 6 patients $(12 \%)$, which was high enough to transfer those patients from the group of moderate injuries to that with mild disorders. In addition, 2 subjects (2\%) from the group with severe injuries were allocated to moderate and mild head injury groups.

The initial VNG examination showed that 19 patients (38\%) had the features of the peripheral vestibular system impairment, 20 patients (40\%) had dysfunction of the central part, and in 11 patients (22\%), the features of both dysfunctions were recorded. After 6 months, the normalization of VNG records was observed in 19 patients (38\%). An improvement was significantly more frequently related to peripheral disorders as compared to central ones (19 subjects vs. $4, \mathrm{p}<0.001)$. In 31 patients $(62 \%)$, features of vestibular dysfunction were still noted. These patients were found to have statistically more frequent central disorders compared to peripheral ones (34\% vs. $8 \%, \mathrm{p}<0.001)$ (Fig. 1$)$.

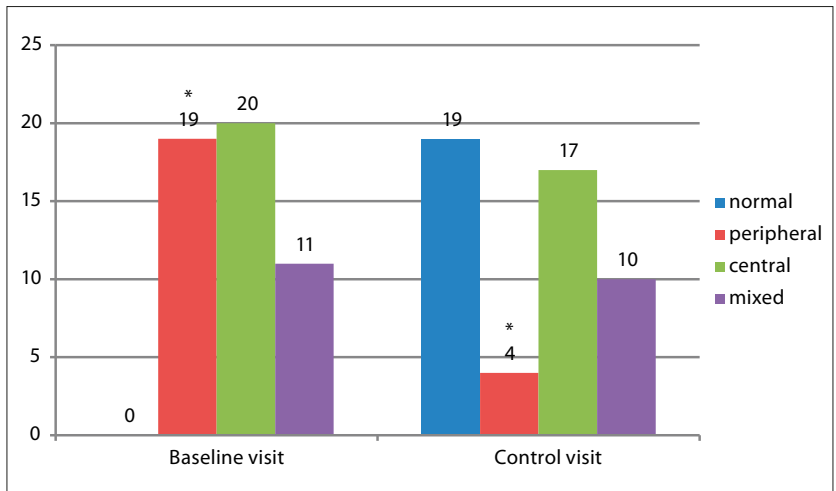

Figure 1. Results of the videonystagmography test in patients after mild traumatic brain injury at baseline visit and 6-month follow-up control visit $(n=50)$

\section{DISCUSSION}

In the presented study, dizziness/vertigo and imbalance were the main symptoms reported by patients after trauma. On the first examination, $40 \%$ of patients stated unsteadiness when walking, periodic spatial orientation disorders, and static imbalance. Those disturbing ailments were the cause of seeking help from an otolaryngologist. Literature reports demonstrate that their prevalence ranges from 14\% - 90\% depending on the accepted criterion $[16,24]$. Such a large variety of data in the literature results from the fact that nonsystemic post-traumatic dizziness manifests itself in a very heterogeneous manner, and is affected by various factors, such as the severity and type of injury, coexisting illnesses, intensity of the ailment, emotional state and psychological type of the patient, as well as his/her willingness to return to work $[1,17,20]$.

After 6 months, non-systemic dizziness was reported by nearly a quarter of patients in the analyzed group. This is not surprising because the literature reports demonstrate that the time of symptoms' assessment and the range of performed tests in patients after injury are very different. Hoffer et al. [25] observed that persistent dizziness and balance instability were the predominant symptoms 3 months after head trauma. Ernst et al. [15], in a group of 63 patients with dizziness who were examined between 3 weeks to 3 months after traumatic injury, found various otoneurological disorders such as perilymphatic fistula and peripheral lesions, including otolithic and central ones.

Patients in the current study subjectively evaluated their symptoms by completing the DHI questionnaire twice. After 6 months, there was a statistically significant decrease in the symptom intensity in all evaluated domains, and hence the transition of patients to the group of moderate and mild disorders was observed. These results coincide with the data provided by Basford et al. [14] who showed significant differences in DHI scores in traumatic patients compared to a control group. Kaufman et al. [21], in 10 patients examined on average 2.8 years after brain injury, found statistically significant correlations between the outcomes of the physical subscale of DHI and post-urography results, as did Lin et al. [17]. Gottshall et al. [26] reported improvement in the results obtained in the DHI questionnaire and dynamic visual acuity examination performed weekly for 4 weeks in patients after mild traumatic head injury.

In the presented study, only those patients were included in whom vestibular impairment was diagnosed in the initial VNG examination. In this test, features of the peripheral and central vestibular dysfunction were identified in approximately $40 \%$ of patients. There is a small number of studies with which these results can be compared. Naguib et al. [18] demonstrated that at the early stage after head injury, central vestibular lesions were more frequently identified in the VNG test than peripheral ones (60\% vs. $24 \%$, respectively). However, the authors investigated patients with different levels of head trauma severity. Marzo et al. [27], while evaluating 16 patients who were diagnosed from $1-6$ months after injury due to imbalance and dizziness, found abnormal results of the balance tests in half of the patients. Suh et al. [19], in the eye-tracking test in patients both at the early stage and after 5 years of a follow-up, observed inter alia impaired tracking predictability, which correlated with cognitive impairment in those patients. Davies and Luxon [13] in their study on 100 patients after head injuries of different levels of severity, including $72 \%$ with mild trauma, observed only a semicircular canal dysfunction in $20 \%$ of patients and central dysfunction in $8 \%$. Basford et al. [14] stated significantly more frequent disturbances in the caloric and optokinetic tests in patients after head traumas. Thus, these results again indicate a very large variation in the population of patients studied. In addition, Fife and Kalra [28], however, emphasize that the absence of disturbances detected in VNG test does not exclude the peripheral cause of ailments that may be related to abnormalities of the utricle and sacculus in patients after brain injury, which was presented in another publication [9]. 
In the present study, the compensation and normalization of the VNG records occurred in almost $40 \%$ of the patients after 6 months, while the central disorders were significantly more frequently identified in the remaining patients. Similarly, Naguib et al. [18] reported the withdrawal of subjective and objective features of the balance disorders evaluated with the use of the VNG test within 3 months after injury.

Patients with balance system dysfunction after head injury constitute a very diverse group, taking into account demographic and clinical considerations. Dizziness and sensation of postural instability usually make the patient seek specialized diagnostic examinations.

\section{CONCLUSIONS}

1. In patients after mild traumatic brain injury, about one fourth of them had vestibular system dysfunction with the same frequency of peripheral, central and mixed lesions in videonystagmography.

2. In patients with vestibular dysfunction after 6 months, intensity of subjective symptoms dependent on vestibular system decreased; however, more than half of these patients still had abnormal VNG results, mainly indicate central pathology.

\section{Acknowledgment}

The study received financial support from the Medical University in Lodz, Poland (Grant No. 503/2-036-02/50321-002).

\section{REFERENCES}

1. Cassidy JD, Cancelliere C, Carroll LJ. Côté P, Hincapié CA, Holm LW, et al. Systematic review of self-reported prognosis in adults after mild traumatic brain injury: results of the International Collaboration on Mild Traumatic Brain Injury Prognosis. Arch Phys Med Rehabil. 2014; 95(3 Suppl): S132-151.

2. Donovan J, Cancelliere C, Cassidy JD. Summary of the findings of the International Collaboration on Mild Traumatic Brain Injury Prognosis. Chiropr Man Therap. 2014; 22(1): 38.

3. Hoffer ME, Schubert MC, Balaban CD. Early diagnosis and treatment of traumatic vestibulopathy and postconcussive dizziness. Neurol Clin. 2015; 33(3): 661-668.

4. Roozenbeek B, Maas AI, Menon D. Changing patterns in the epidemiology of traumatic brain injury. Nat Rev Neurol. 2013; 9(4): 231-236.

5. Thurman DJ, Alverson C, Dunn KA, Guerrero J, Sniezek JE. Traumatic brain injury in the United States: A public health perspective. J Head Trauma Rehabil. 1999; 14(6): 602-615.

6. Nygren-de Boussard C, Holm LW, Cancelliere C, Godbolt AK, Boyle E, Stålnacke BM, et al. Nonsurgical interventions after mild traumatic brain injury: a systematic review. Results of the International Collaboration on Mild Traumatic Brain Injury Prognosis. Arch Phys Med Rehabil. 2014; 95(3 Suppl): S257-264.
7. Ruff RM, Iverson GL, Barth JT. Bush SS, Broshek DK; NAN Policy and Planning Committee. Recommendations for diagnosing a mild traumatic brain injury: a National Academy of Neuropsychology education paper. Arch Clin Neuropsychol. 2009; 24(1): 3-10.

8. Jennet B. Epidemiology of head injury. J Neurol Neurosurg Psychiatry. 1996; 60(4): 362-369.

9. Józefowicz-Korczyńska M, Skóra W, Pajor A. Benign paroxysmal positional vertigo in patients after mild traumatic brain injury. Adv Clin Exp Med. in press

10. Liu H. Presentation and outcome of post-traumatic benign paroxysmal positional vertigo. Acta Otolaryngol. 2012; 132(8): 803-806.

11. Motin M, Keren O, Groswasser Z, Gordon CR. Benign paroxysmal positional vertigo as the cause of dizziness in patients after severe traumatic brain injury: diagnosis and treatment. Brain Inj. 2005; 19(9): 693-697.

12. Chamelian L, Feinstein A. Outcome after mild to moderate traumatic brain injury: the role of dizziness. Arch Phys Med Rehabil. 2004; 85(10): 1662-1666.

13. Davies RA, Luxon LM. Dizziness following head injury: a neurootological study. J Neurol. 1995; 242(4): 222-230.

14. Basford JR, Chou LS, Kaufman KR, Brey RH, Walker A, Malec JF, et al. An assessment of gait and balance deficits after traumatic brain injury. Arch Phys Med Rehabil. 2003; 84(3): 343-349.

15. Ernst A, Basta D, Seidl RO, Todt I, Scherer H, Clarke A. Management of posttraumatic vertigo. Otolaryngol Head Neck Surg. 2005; 132(4): 554-558.

16. Hoffer ME, Gottshall KR, Moore R, Balough BJ, Wester D. Characterizing and treating dizziness after mild head trauma. Otol Neurotol. 2004; 25(2): 135-138.

17. Lin LF, Liou TH, Hu CJ, Ma HP, Ou JC, Chiang YH, et al. Balance function and sensory integration after mild traumatic brain injury. Brain Inj. 2015; 29(1): 41-46.

18. Naguib MB, Madian Y, Refaat M, Mohsen O, El Tabakh M, Abo-Setta A. Characterisation and objective monitoring of balance disorders following head trauma, using videonystagmography. J Laryngol Otol. 2012; 126(1): 26-33.

19. Suh M, Kolster R, Sarkar R, McCandliss, Ghajar J. Deficits in predictive smooth pursuit after mild traumatic brain injury. Neurosci Lett. 2006; 401(1-2): 108-113.

20. Szczupak M, Hoffer ME, Murphy S, Balaban CD. Posttraumatic dizziness and vertigo. Handb Clin Neurol. 2016; 137: 295-300.

21. Kaufman KR, Brey RH, Chou LS, Rabatin A, Brown AW, Basford JR. Comparison of subjective and objective measurements of balance disorders following traumatic brain injury Med Eng Phys. 2006; 28(3): 234-239.

22. Wallace B, Lifshitz J. Traumatic brain injury and vestibulo-ocular function: current challenges and future prospects. Eye and Brain 2016; 8: 153-164.

23. Jacobson GP, Newman CW: The development of the Dizziness Handicap Inventory. Arch Otolaryngol Head Neck Surg. 1990; 116(4): 424-427.

24. Lei-Rivera L, Sutera J, Galatioto JA, Hujsak BD, Gurley JM. Special tools for the assessment of balance and dizziness in individuals with mild traumatic brain injury. Neuro Rehabilitation. 2013; 32(3): 463-472.

25. Hoffer ME, Balough BJ, Gottshall KR. Posttraumatic balance disorders. Int Tinnitus J. 2007; 13(1): 69-72.

26. Gottshall K, Drake A, Gray N, McDonald E, Hoffer ME. Objective vestibular tests as outcome measures in head injury patients. Laryngoscope 2003; 113(10): 1746-1750.

27. Marzo SJ, Leonetti JP, Raffin MJ, Letarte P. Diagnosis and management of post-traumatic vertigo. Laryngoscope 2004; 114(10): 1720-1723.

28. Fife TD, Kalra D. Persistent vertigo and dizziness after mild traumatic brain injury. Ann N Y Acad Sci. 2015; 1343: 97-105. 